\title{
Universal dynamics and controlled switching of dissipative Kerr solitons in optical microresonators
}

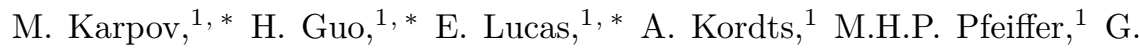 \\ Lihachev,$^{2,3}$ V.E. Lobanov, ${ }^{3}$ M.L. Gorodetsky, ${ }^{2,3}$ and T.J. Kippenberg ${ }^{1, \dagger}$ \\ ${ }^{1}$ École Polytechnique Fédérale de Lausanne (EPFL), CH-1015 Lausanne, Switzerland \\ ${ }^{2}$ Faculty of Physics, M.V. Lomonosov Moscow State University, 119991 Moscow, Russia \\ ${ }^{3}$ Russian Quantum Center, Skolkovo 143025, Russia
}

(Dated: January 20, 2016)

\begin{abstract}
Dissipative temporal Kerr solitons in optical microresonators enable to convert a continuous wave laser into a train of femtosecond pulses. Of particular interest are single soliton states, whose sech ${ }^{2}$ spectral envelope provides a spectrally smooth and low noise optical frequency comb, and that recently have been generated in crystalline, silica, and silicon-nitride resonators. They constitute sources that are unique in their ability to provide short femtosecond pulses at microwave repetition rates. Likewise, they provide essential elements to realize chip-scale, integrated frequency combs for time-keeping, spectroscopy, navigation or telecommunications. However to date, the dynamics of this class of solitons in microresonators remains largely unexplored, and the reliable generation of single soliton states remains challenging. Here, we study the dynamics of multiple soliton states containing $N$ solitons and report the discovery of a novel, yet simple mechanism which makes it possible to reduce deterministically the number of solitons, one by one, i.e. $N \rightarrow N-1 \rightarrow \ldots \rightarrow 1$. By applying weak phase modulation, we directly characterize the soliton state via a double-resonance response. The dynamical probing demonstrates that transitions occur in a predictable way, and thereby enables us to map experimentally the underlying multi-stability diagram of dissipative Kerr solitons. These measurements reveal the "lifted" degeneracy of soliton states as a result of the power-dependent thermal shift of the cavity resonance (i.e. the thermal nonlinearity). The experimental results are in agreement with theoretical and numerical analysis that incorporate the thermal nonlinearity. By studying two different microresonator platforms (integrated $\mathrm{Si}_{3} \mathrm{~N}_{4}$ microresonators and crystalline $\mathrm{MgF}_{2}$ resonators) we confirm that these effects have a universal nature. Beyond elucidating the fundamental dynamical properties of dissipative Kerr solitons the observed phenomena are also of practical relevance, providing a manipulation toolbox which enables to sequentially reduce, monitor and stabilize the number $N$ of solitons, preventing it from decay. Achieving reliable single soliton operation and stabilization in this manner in optical resonators is imperative to applications.
\end{abstract}

Microresonator frequency combs (Kerr combs) have opened a novel research area at the interface of microand nano-photonics and frequency metrology [1, 2]. Kerr combs are generated in high- $Q$ millimeter- or micronscale resonators via parametric processes driven by continuous wave $(\mathrm{CW})$ laser [3, 4]. Kerr combs have attracted significant attention over past years due to unprecedented compactness, demonstrated octave-spanning operation [5, 6], repetition rates in the microwave domain (> $10 \mathrm{GHz}$ ), and ability to be operated in low noise regimes [7 9]. They promise chip-scale optical frequency combs connecting RF to optical domain that could make metrology ubiquitous, widely accessible beyond specialized metrology laboratories. Recently, it has been demonstrated that Kerr combs can be operated in the regime of temporal dissipative Kerr solitons (DKS) 10, 11. DKS allow for fully coherent optical frequency combs (soliton combs) that can be sufficiently broadband for self-referencing via soliton induced Cherenkov radiation [11, and provide access to stable ultrashort pulses of tunable duration [10, 12 at microwave repetition rates [13. Of particular interest are single soliton states, that exhibit a spectrally smooth sech ${ }^{2}$ envelope. Such soliton based frequency comb sources have a wide range of appli- cations including molecular spectroscopy [14], coherent data transmission [15, 16], arbitrary waveform generation [8, optical clocks [17] or astrophysics [2], and more generally in applications where short pulse duration at microwave repetition rate is desirable.

Originally discovered to spontaneously form in crystalline $\mathrm{MgF}_{2}$ resonators [10] (and for the first time externally induced in optical fiber cavities [18]), DKS have been demonstrated in a variety of high- $Q$ resonator platforms, ranging from silica wedge resonators [13], to $\mathrm{Si}_{3} \mathrm{~N}_{4}$ photonic chips [11] and compact crystalline resonators pumped via distributed feedback lasers [19, 20]. Due to the recent nature of these findings, the soliton formation process and its dynamics remain to date largely unexplored. While solitons have been reported in a number of platforms, the soliton generation procedures in high$Q$ microresonators are inherently stochastic (techniques used in optical fiber cavities 21 are technically impractical due to much shorter round-trip time of microresonators). While CW laser tuning and "power kicking" schemes were proposed [11, 13] for soliton generation, these techniques presently do not allow to control the number of solitons formed in the resonator. Another important question is the possibility of deterministic ma- 
nipulation of states with multiple solitons in microresonators. Even though the states with various number of solitons could be generated in optical microresonators, the transitions between them take place stochastically via pairwise interactions of solitons when the pump is tuned, and cannot be predicted so far. Due to these effects, deterministic generation of the single soliton state still represents an outstanding challenge. One more challenge is the non-destructive monitoring of the soliton state. The soliton regime in microresonators is fragile (though self-sustainable) and is not persistent against significant thermal drifts and other external perturbations. The reported passive lifetime of DKS achieves several hours in a stable laboratory environment [10, 11], however, no technique is known to enable feedback stabilized control of soliton state, preventing it from decay.

In this paper we report the discovery of a phenomenon that allows to induce deterministically transitions to states with less solitons (i.e. from $N$ to $N-1$ ), and thereby to reliably reach the single soliton state. The phenomenon is not explained by standard theoretical simulations based on the Lugiato-Lefever equation (LLE) or coupled mode equations models [10, 22, 26]. We present detailed analysis of the observed phenomenon, in two microresonator platforms where the thermal locking is possible, and demonstrate its universal nature. The reported findings allow to switch between multiple-soliton states by sequentially reducing the number $N$ of initially created solitons (with a routine simple enough to be carried out by a micro-controller), to monitor and control the switching, and to hold the targeted soliton state, preventing it from decay. Especially, the single soliton state can be deterministically and reliably induced, which is imperative to a wide range of applications. The presented results contribute to the physical understanding of switching behavior of the DKS, highlight the influence of thermal effects and provide a rich toolbox for the study of the multiple-soliton dynamics. From an applied perspective, the results present a route to making reliable pulse sources and frequency combs based on DKS at microwave repetition rates in optical microresonators.

\section{RESULTS}

Observation of switching between dissipative Kerr soliton states by laser backward tuning. The principles of microresonator frequency comb generation and the formation of dissipative Kerr solitons (DKS) are shown in Fig.1(a). CW laser light is coupled to a high- $Q$ optical resonator, where modulation instability (MI) and cascaded four-wave-mixing processes lead to the formation of a broadband frequency comb. In this work we study two microresonator platforms: $\mathrm{Si}_{3} \mathrm{~N}_{4}$ on-chip ring microresonators [11, 27, 29] (Fig]1(b)) and $\mathrm{MgF}_{2}$ crystalline resonators [10, 30-32] (Fig]1.c)). The laser tun- ing technique was developed as an effective method to the formation of dissipative Kerr solitons [10], in which the CW pump laser is tuned (from short to long wavelengths) over the cavity resonance, referred to as the "forward tuning". Initially, the CW pump is in the bluedetuned regime. The cavity resonance is shifted due to the slow thermal and fast Kerr nonlinearity of the microresonator, resulting in a self-locking of the cavity resonance to the pump laser [33, 34. In this regime the Kerr comb formation can be observed. The mechanism results in a triangular trace in the generated comb light, over the pump frequency detuning. When the pump is tuned over the cavity resonance, it enters the effectively red-detuned regime where multiple dissipative Kerr solitons (i.e. multiple-solitons) can be formed. The soliton state is accompanied with a step-like power trace in the generated comb light, where the step height corresponds to the number of solitons $(N)$ inside the resonator. Transitions to states with lower number of solitons may also occur and the power trace will exhibit a characteristic steps [10. Eventually, by stopping the pump laser tuning at a step while ensuring the thermal equlibrium in the resonator, stable multiple-soliton and even single soliton states can be accessed (Fig, 1(d)). This forward tuning method was applied in $\mathrm{MgF}_{2}, \mathrm{Si}_{3} \mathrm{~N}_{4}$ and silica resonators for single dissipative Kerr soliton generation [10, 11, 35].

However, in on-chip microresonators, the thermal nonlinearity significantly impacts the soliton step pattern, such that single soliton states become rarely accessible with the forward tuning method. Figure 1(e) shows 200 overlaid experimental power traces of the generated comb light obtained in a $\mathrm{Si}_{3} \mathrm{~N}_{4}$ microresonator in the forward tuning, in which only multiple-soliton states are stochastically accessed having $N=6$ (predominantly), 7, 8 or 9. Careful studies further reveal several common features in $\mathrm{Si}_{3} \mathrm{~N}_{4}$ microresonators, irrespective of the employed pump power (see Supplementary Information (SI) for more details) and the laser tuning speed: (1) the distributions mostly consist of the traces with one step corresponding to high- $N$ multiple-soliton state; (2) the accessible step length decreases with decreasing $N$; (3) the number of generated solitons increases with increasing pump power. All of these imply that the single soliton state is not readily accessible in the forward tuning.

Remarkably, an additional laser tuning towards shorter wavelengths ("backward tuning") provides a way to reliably access the single soliton state starting from an arbitrary multiple-soliton state. The result of this backward tuning sequence, shown in Fig.1(f), allows for successive extinction of intracavity solitons (soliton switching) down to the single soliton state $(N \rightarrow N-1 \rightarrow \ldots \rightarrow 1)$. Figure $1(\mathrm{~g})$ shows one trace of the generated light of the $\mathrm{Si}_{3} \mathrm{~N}_{4}$ microresonator, where switching from seven solitons to the single soliton is observed. Strikingly, the power trace of the generated comb light reveals a regular staircase pattern with equal stair length and height. The exact 
a

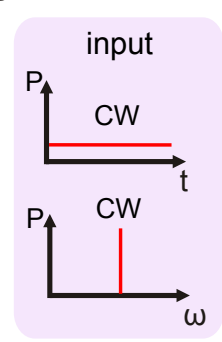

d

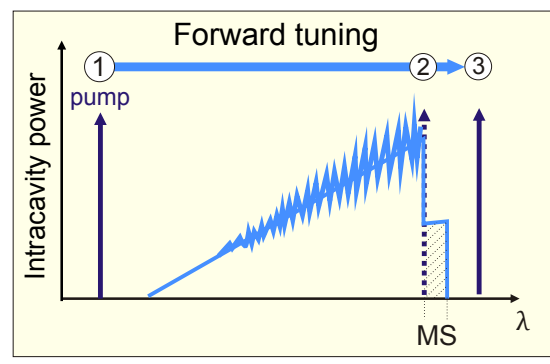

f
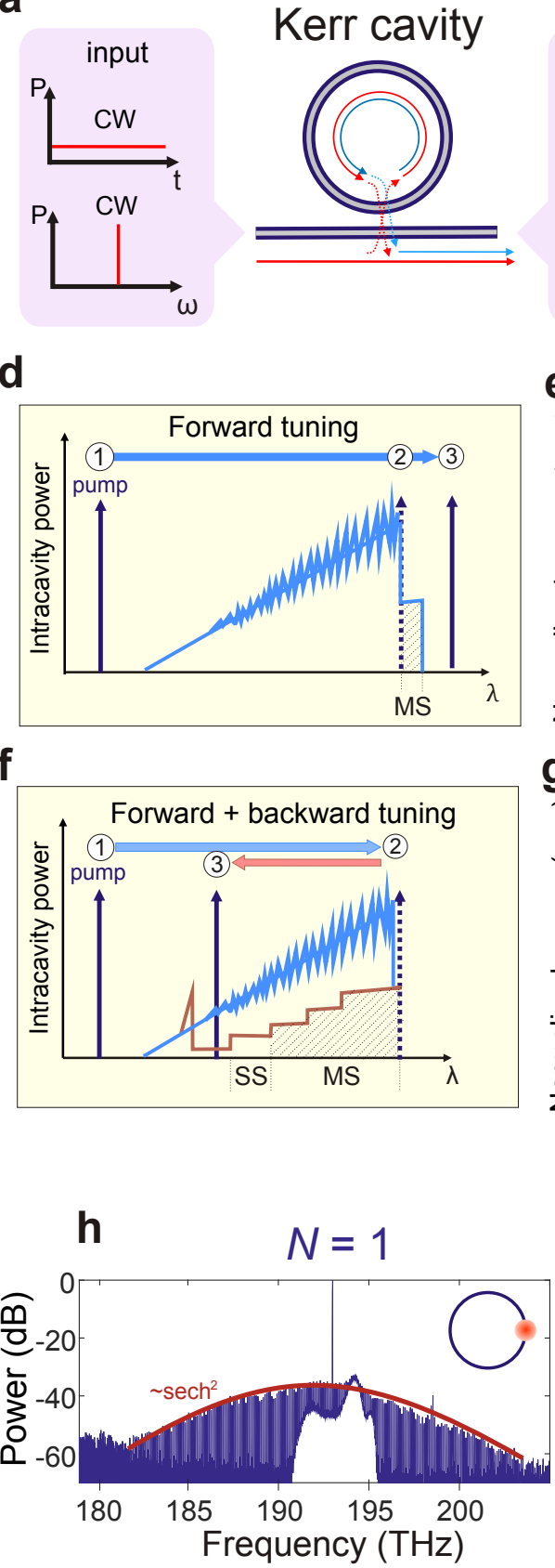

b
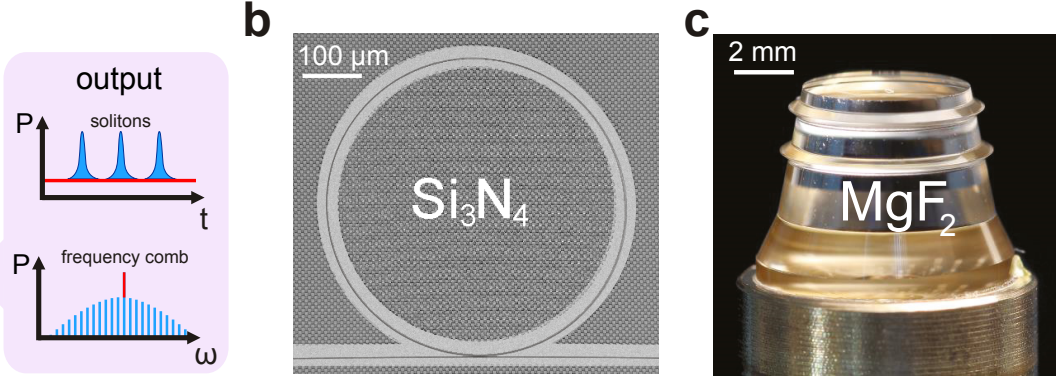

e

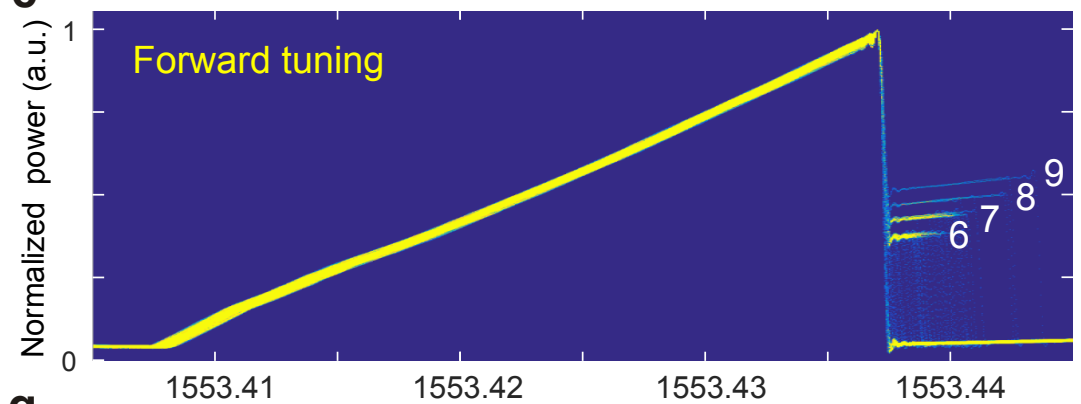

g

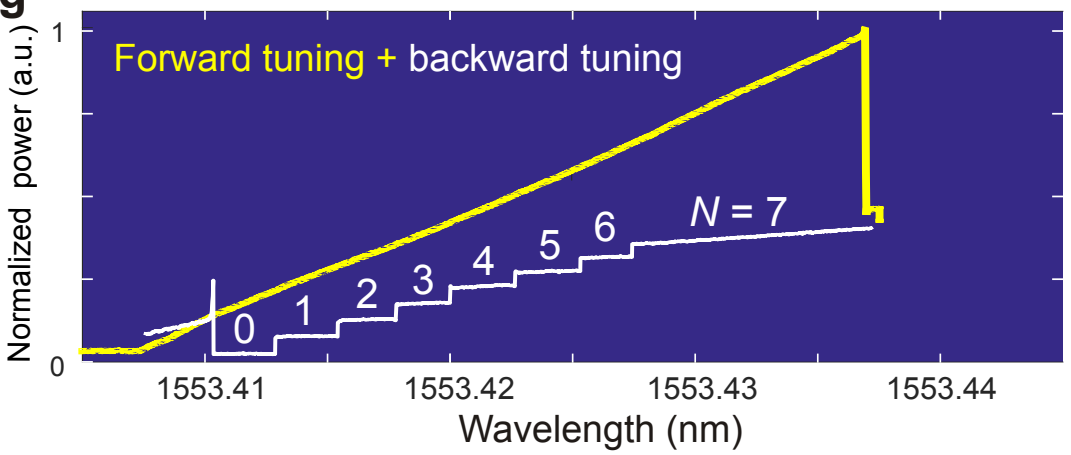

i

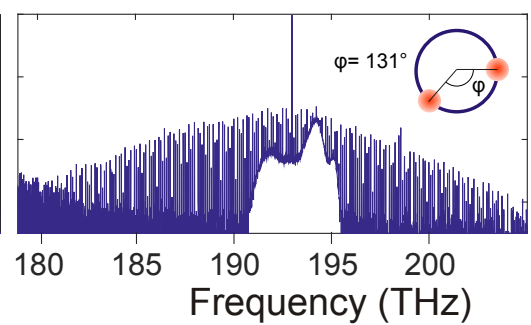

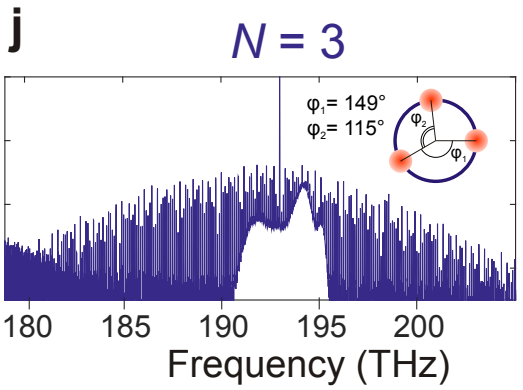

Figure 1. Forward and backward tuning of the pump. (a) The principle of microresonator frequency comb generation and the formation of dissipative Kerr solitons; (b) SEM image of the $\mathrm{Si}_{3} \mathrm{~N}_{4}$ on-chip microresonator with a free spectral range (FSR) of $100 \mathrm{GHz}$; (c) Picture of the $\mathrm{MgF}_{2}$ crystalline resonator with FSR of $14 \mathrm{GHz}$; (d) Scheme of the laser tuning method for the soliton generation in optical microresonators. The pump laser is tuned over the resonance from short to long wavelengths (forward tuning). Hatched region indicates the pump detuning range of multiple solitons (MS); (e) Histogram plot of 200 overlaid experimental traces of the output comb light in the pump forward tuning over the resonance with the same pump power and tuning speed, which reveals the formation of a predominant multiple soliton state with $N=6$; The noise pattern in the forward detuning was not captured by the measurements due to the averaging in the photodetector; (f) Scheme of the laser backward tuning. To initiate the sequence, the forward tuning is first applied, and the pump is stopped in a multiple soliton state (which can be stable by suitable choice of the laser tuning speed). In the second stage, the pump is tuned back to short wavelengths, which leads to successive soliton switching, $N \rightarrow N-1 \rightarrow \ldots \rightarrow 1$. The MS area indicates the detuning range of multiple soliton states, which is much larger compared to the forward tuning method. There also exists the range of the single soliton state (SS); (g) Experimental trace in the forward tuning (yellow curve) followed by one trace in the backward tuning (white curve) with successive transitions of multiple-soliton states from $N=7$ to $N=0$ (no solitons); (h-j) frequency comb spectra in soliton states with $N=1,2,3$, measured during the backward tuning in a $100 \mathrm{GHz}^{\mathrm{Si}} \mathrm{N}_{3} \mathrm{~N}_{4}$ microresonator; 
soliton number in each step can be precisely inferred from the step height. The pattern is almost identical over multiple experimental runs (using the same tuning speed and pump power) regardless of the initial soliton number $N$. Each transition between multiple-soliton states occurs with the extinction of preferably one soliton at a time, which is confirmed by the relative positions of the intracavity solitons that are retrieved from the optical spectrum (cf. insets in Fig $1(h-j)$ ).

In experiments, the backward tuning process must be adiabatic to induce the successive reduction of the soliton number: the thermal equilibrium is required at each multiple-soliton state. This prerequisite is satisfied by choosing a tuning speed much slower than the thermal relaxation rate that depends on the effective mode volume and the thermal diffusivity of a microresonator [36]. For the employed $\mathrm{Si}_{3} \mathrm{~N}_{4}$ microresonator the backward tuning speed is chosen $\sim 40 \mathrm{MHz} / \mathrm{s}$, while the forward tuning speed is $\sim 100 \mathrm{GHz} / \mathrm{s}$. In this way all soliton states $(\leq N)$ are deterministically accessible. In contrast to the robust backward tuning that enables successive extinction of intracavity solitons, the forward tuning in $\mathrm{Si}_{3} \mathrm{~N}_{4}$ microresonators always leads to collective extinction of solitons.

The backward tuning was also studied in $\mathrm{MgF}_{2}$ crystalline microresonators, where the successive soliton switching to the single soliton state is also achieved. In contrast to the $\mathrm{Si}_{3} \mathrm{~N}_{4}$ platform, the single soliton state can directly be accessed with the forward tuning in $\mathrm{MgF}_{2}$ microresonators [10]. Yet, this requires fine adjustments on the coupling, the pump power and the tuning speed. The backward tuning, on the other hand, is much more robust and significantly facilitates the generation of single soliton states for crystalline resonators.

The soliton switching in both $\mathrm{Si}_{3} \mathrm{~N}_{4}$ and crystalline $\mathrm{MgF}_{2}$ resonator, proves that the backward tuning represents a universal approach to the generation of a single soliton state in microresonators, provided that the thermal locking can be achieved.

Non-destructive probing of the soliton response. Dissipative Kerr solitons in microresonators represent stable and self-reinforcing intracavity light patterns resulting from double balance between pump and cavity losses, as well as chromatic dispersion and Kerr nonlinearity of the resonator. The key parameter of such soliton state is the effective laser frequency detuning that determines both the amplitude and the duration of soliton pulses [10]. This detuning is defined as $2 \pi \delta_{\text {eff }}=\widetilde{\omega}_{0}-\omega_{\mathrm{p}}$, where $\widetilde{\omega}_{0}$ indicates the frequency of a cavity resonance and $\omega_{\mathrm{p}}$ is the pump laser frequency. In experiments the pump frequency is precisely controlled, but the resonance frequency is thermally shifted from the initial cold cavity resonance frequency $\omega_{0}$, making it $a$ priori not possible to evaluate the effective detuning. On the other hand, the absolute detuning $2 \pi \delta=\omega_{0}-\omega_{\mathrm{p}}$ can be introduced and measured as the position of the pump frequency relative to the fixed cold cavity resonance. It has been shown that solitons are supported within a certain range of the effective detuning [10, 12, when the pump is effectively-red detuned $\left(\omega_{\mathrm{p}}<\widetilde{\omega}_{0}\right)$, which we refer to as the soliton existence range for a given constant input power.

We developed a non-destructive soliton probing scheme that allows to track the effective detuning and extract the soliton number $N$ of microresonator frequency combs. The setup, presented in Fig,2(a), employs a pump laser, whose frequency is phase modulated using a vector network analyzer (VNA), that produces weak optical sidebands with sweeping frequency $(\nu)$ in the range $5 \mathrm{kHz}-$ $4.5 \mathrm{GHz}$, which probe the state of the microresonator system. The complex modulation response to such probes is measured by the VNA.

This probing method enables to identify different stages in the generation of frequency comb, including the soliton formation, see Fig,2(b). First, when the pump is in the blue-detuned regime $\left(\omega_{\mathrm{p}}>\widetilde{\omega}_{0}\right)$, away from the cavity resonance, the modulation response on the VNA shows a Lorenzian-like resonance profile that corresponds to the cavity resonance with the peak position indicating $\left|\delta_{\text {eff }}\right|$. Second, when (forward) tuning the pump frequency into the cavity resonance, where the frequency comb in the chaotic MI regime is observed, the modulation response shows an asymmetric profile with the peak position being fixed, indicating the thermal and Kerr locking of the cavity resonance to the pump frequency. Third, when the frequency comb is in the soliton state, with the pump laser tuned in the soliton existence range in the red-detuned regime, the modulation response shows unexpectedly a double-resonance feature. Finally, when the pump frequency is tuned out of the soliton existence range where no comb is observed, the modulation response shows again a single, Lorenzian-like resonance similar to the first stage.

The double-resonance response that is observed in the presence of soliton states can be attributed to the superposition of weak continuous background and intense soliton pulses [10]. Due to different intensities each component induces a different Kerr shift to the cavity resonance which we can discriminate by the modulation probing. Since the pump is far detuned from the cavity resonance, the high-frequency peak in the modulation response corresponds to the cavity resonance that is slightly shifted by the $\mathrm{CW}$ component ( $\mathcal{C}$-resonance). The peak position in this way indicates the effective detuning. On the other hand, the resonance shifted by solitons appears as the low-frequency peak ( $\mathcal{S}$-resonance). The position of the $\mathcal{S}$-resonance is nearly fixed as it depends on the intensity of individual soliton, while the magnitude is related to the number of solitons $(N)$.

We applied the non-destructive soliton probing to both $\mathrm{Si}_{3} \mathrm{~N}_{4}$ and $\mathrm{MgF}_{2}$ microresonators. The double-resonance response is observed in both platforms when having soli- 

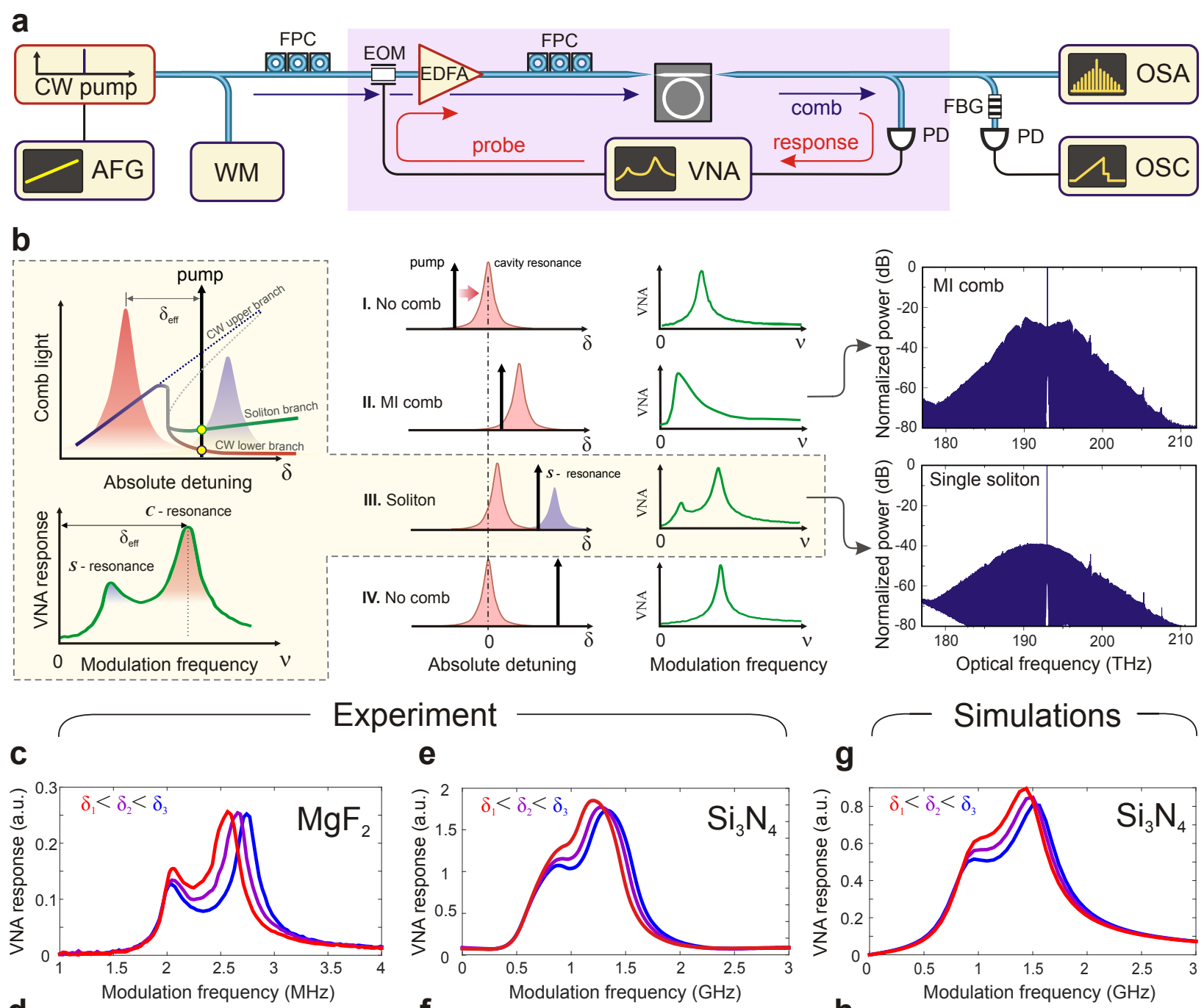

Experiment
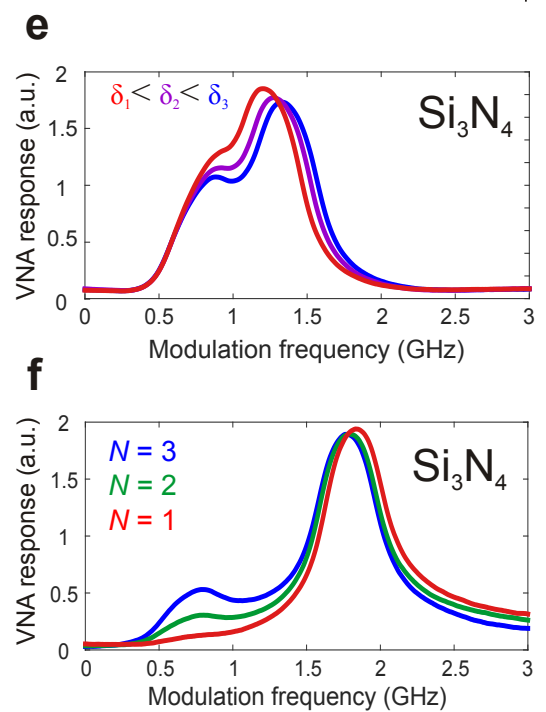

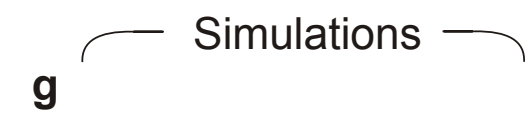

h
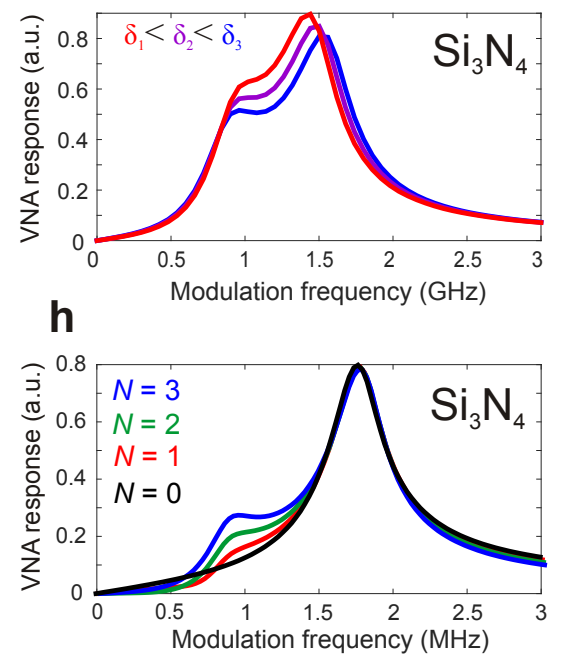

Figure 2. Dynamical probing of dissipative temporal Kerr solitons (DKS) in microresonators. (a) Setup scheme used for soliton generation, non-destructive soliton probing and deterministic soliton switching. An external cavity diode laser (CW pump) is used as a pump source. AFG, arbitrary function generator; EDFA, erbium doped amplifier; FPC, fiber polarization controller; WM, wavelength meter; VNA, vector network analyzer; OSA, optical spectrum analyzer; OSC, oscilloscope; PD, photodiode; EOM, electro-optical phase modulator; PM, phase modulator; FBG, fiber Bragg grating. (b) (Left, top) Diagram of the double-resonance modulation response in the soliton state. The green line indicates the power trace of the soliton component which is evolved from the high-intensity branch of the bistability (blue line). The pump is tuned in the bistability range (in the effective-red detuned regime). Therefore, both the soliton branch and the low-intensity continuous (CW) branch (red line) are supported in the system, each corresponds to a resonance, i.e. the $\mathcal{S}$-resonance and the $\mathcal{C}$-resonance. (Left, down) Double-resonance modulation response from VNA. The high-frequency peak indicates the $\mathcal{C}$ resonance and the lowfrequency is the $\mathcal{S}$-resonance. (Middle) Four stages of the microresonator frequency comb formation and corresponding VNA modulation response when the pump laser is forward tuned over the resonance: (I) No comb, the pump is blue-detuned; (II) Chaotic MI comb state; (III) Soliton state; (IV) No comb, the pump is red-detuned. (right) Frequency comb spectra corresponding to the chaotic MI operation regime and the single soliton state. (c-f) Experimental double-resonance response of various multiple-soliton states at different detunings for $\mathrm{MgF}_{2}$ and $\mathrm{Si}_{3} \mathrm{~N}_{4}$ microresonators; (i-j) Simulated double-resonance response for $\mathrm{Si}_{3} \mathrm{~N}_{4}$ microresonator used in the work. 
a

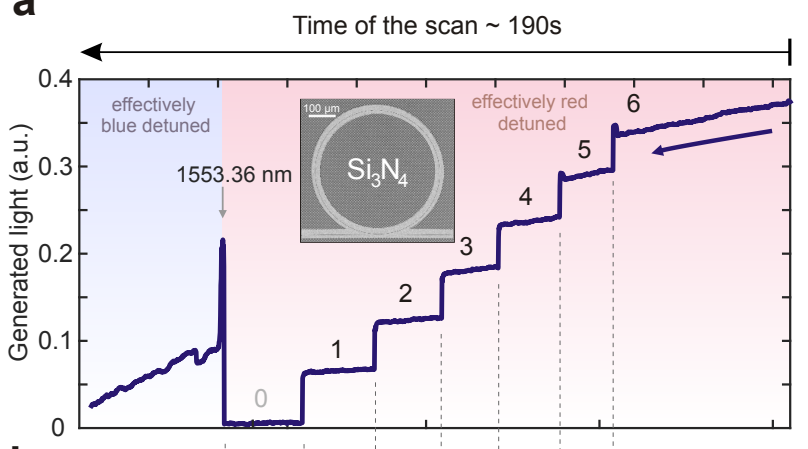

b

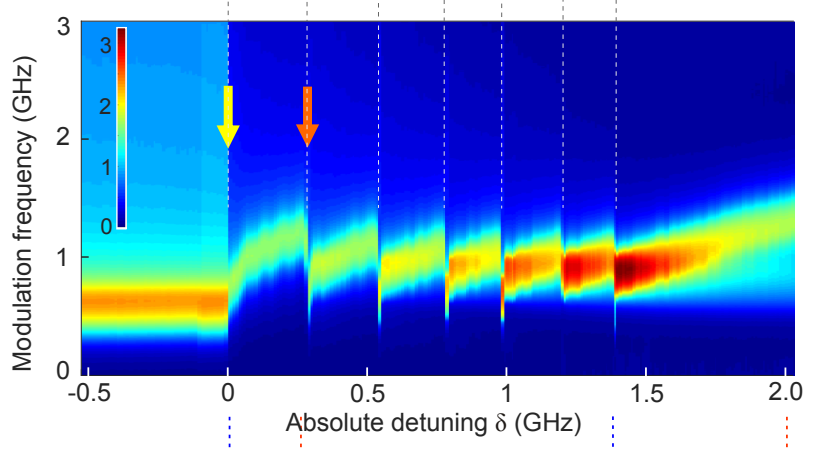

C

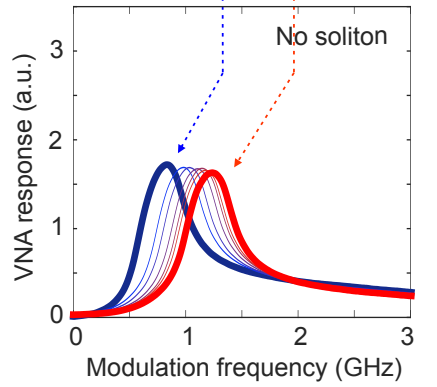

d

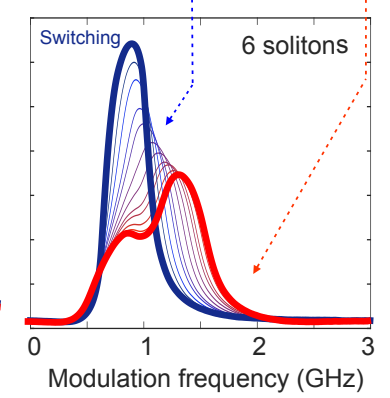

e

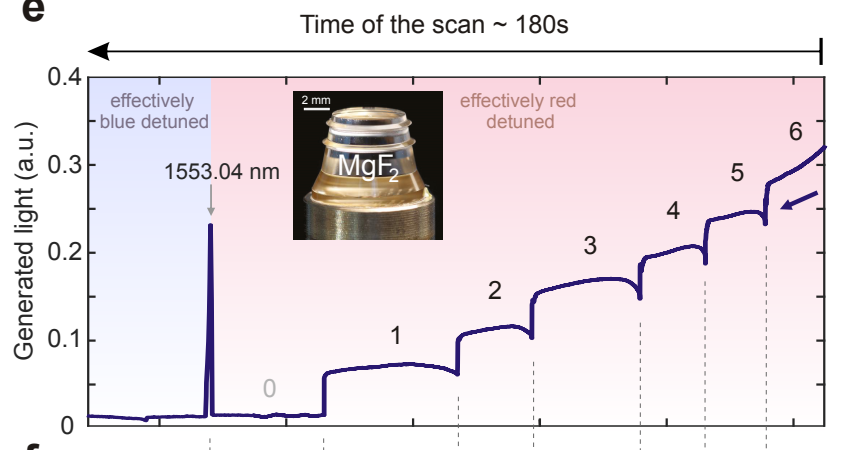

f

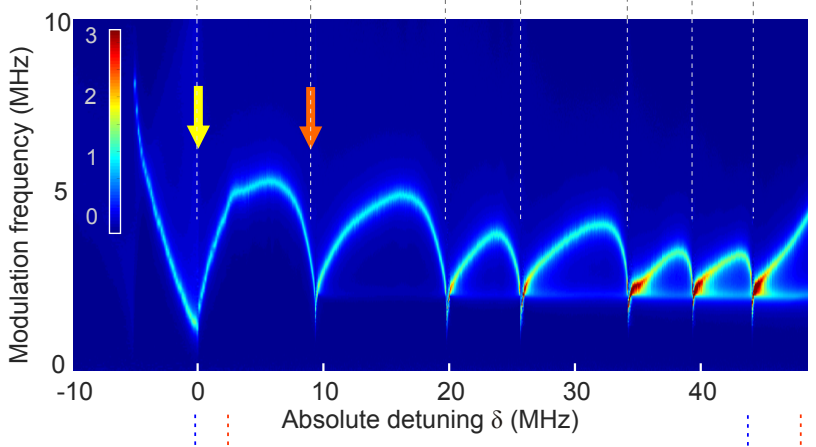

$\mathbf{h}$

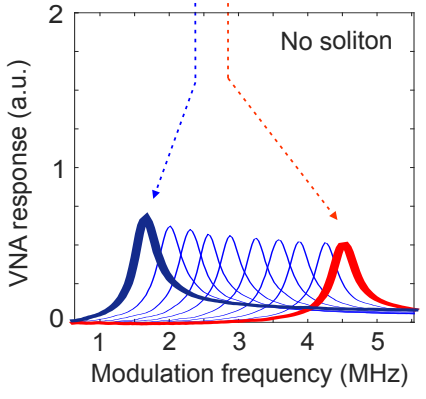

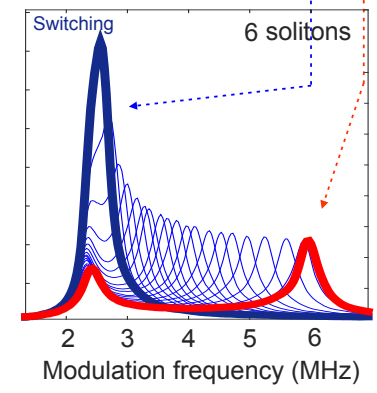

Figure 3. Deterministic switching of the soliton states. (a) The power trace of the generated light obtained from 100 $\mathrm{GHz} \mathrm{Si}_{3} \mathrm{~N}_{4}$ microresonator with the backward pump tuning from multiple-soliton with $N=6$ (effectively red detuned) to the effectively blue detuned regime; (b) Set of 500 concatenated VNA traces that were taken during the backward tuning shown in (a). The orange arrow indicates the transition from a single soliton state to no-soliton state, while the pump is still red detuned with respect to the cavity resonance. The yellow arrow indicates the transition from the red detuned operating regime to the blue detuned regime; (c) Evolution of the modulation response during the backward tuning process in the effectively red detuned regime, with no soliton presented $(N=0)$; (d) Evolution of the modulation response in the multiple-soliton state with $N=6$; (e) The power trace of the generated light obtained from $14 \mathrm{GHz}_{\mathrm{MgF}}$ crystalline resonator with the backward pump tuning from multiple-soliton state with $N=6$ (effectively red detuned) to the effectively blue detuned regime; (f) Set of $\sim 1700$ concatenated VNA traces that were taken during the backward tuning shown in (e); (g) Evolution of the modulation response during backward tuning in the state with no soliton presented; (h) Evolution of modulation response in the multiple-soliton state with $N=6$.

ton state frequency combs, and is investigated with different soliton number $N$ and pump detunings, see Fig.2(cf). The response is qualitatively similar for both platforms. The peak position of the $\mathcal{C}$-resonance varies with the pump frequency $(\operatorname{Fig} 2(\mathrm{~d}, \mathrm{e}))$, while the $\mathcal{S}$-resonance frequency is practically fixed as predicted. The peak height of the $\mathcal{S}$-resonance linearly depends on the soliton number $N($ Fig, 2(d, f)). We also performed a theoretical analysis of the non-destructive soliton probing scheme, which confirms the double-resonance response of a soliton state (see Fig.2(g, h)).

The response of dissipative Kerr solitons to weak amplitude pump modulation was earlier numerically investigated in [37. While two peaks in the response were also numerically observed in that work (and attributed conceptually to Feshbach and relaxation oscillations in the 
presence of third order dispersion), the present work reveals the underlying physical origin of the soliton probing scheme, not requiring higher order dispersion. Moreover, phase modulation provides higher contrast of the modulation response.

As a way to extract the effective pump detuning $\delta_{\text {eff }}$, the probing technique enables to precisely track the process of microresonator frequency comb generation in experiments. In a soliton state, thermal drifts of the cavity resonance originating from various external sources may cause variations of $\delta_{\text {eff }}$. Based on the modulation response, the effective detuning can be monitored and adjusted (e.g. by tuning the pump frequency) in order to maintain the soliton state within the soliton existence range. In practice, feedback-locking of $\delta_{\text {eff }}$ is possible, which allows for long-term operation of a soliton state in a microresonator.

Deterministic switching of soliton states. We next investigate the transitions of soliton states in the laser backward tuning by applying the non-destructive soliton probing in $\mathrm{Si}_{3} \mathrm{~N}_{4}$ microresonators. We first employ forward tuning in order to generate a multiplesoliton state with $N=6$, and then we perform the slow backward tuning. The power trace of the generated light in the microresonator again shows the staircase pattern in the backward tuning, which corresponds to successive soliton switching from $N=6$ to the single soliton state (Fig,3(a)). The VNA traces are simultaneously recorded and continuously stacked in order to monitor the evolution of the modulation response during the process (see Fig. 3(b)).

The experiments reveal a relationship between the evolution of modulation response and the soliton switching. Within each soliton step, the $\mathcal{C}$-resonance shifts towards the $\mathcal{S}$-resonance due to the decrease of the effective detuning when the laser is tuned backward. When the two resonances overlap, the amplitude of $\mathcal{S}$-resonance is significantly enhanced, leading to a high-intensity singlepeak profile $(\mathrm{Fig} 3(\mathrm{~d}))$. The phenomenon is also confirmed by the theory (cf. SI). The next moment after having such a response, soliton switching occurs, which results in the power drop in the generated light trace as one soliton is extinct $(N \rightarrow N-1)$. After the switching, the $\mathcal{C}$-resonance abruptly separates from the $\mathcal{S}$-resonance. Meantime, while still being Kerr locked, the $\mathcal{S}$-resonance intensity is reduced to a lower level than the previous state, since the number of solitons is reduced by one. In the absence of solitons $(N=0)$, the $\mathcal{S}$-resonance equally is absent in the modulation response, but the $\mathcal{C}$-resonance is still present and captured (Fig, $2(\mathrm{c}))$.

The same measurement was carried out in $\mathrm{MgF}_{2}$ resonators, see Fig, $3(\mathrm{e}-\mathrm{h})$. Similar switching dynamics as in $\mathrm{Si}_{3} \mathrm{~N}_{4}$ microresonators are observed: (1) the power trace shows staircase profile of successive soliton switching; (2) the backward tuning shifts the VNA $\mathcal{C}$-resonance towards the $\mathcal{S}$-resonance; (3) soliton switching occurs with the overlap of $\mathcal{C}$ - and $\mathcal{S}$-resonances and the enhancement of the $\mathcal{S}$-resonance intensity. However, there are several details which differ between $\mathrm{Si}_{3} \mathrm{~N}_{4}$ and $\mathrm{MgF}_{2}$ platforms. First, the optical quality factor $Q$ of $\mathrm{MgF}_{2}$ crystalline resonators $\left(\sim 10^{9}\right)$ is three order of magnitude higher than for $\mathrm{Si}_{3} \mathrm{~N}_{4}$ micro-rings $\left(\sim 10^{6}\right)$. The $\mathcal{C}$ - and $\mathcal{S}$-resonances in the modulation response of crystalline resonator are better resolved as a result of the narrower linewidth. The laser tuning range in $\mathrm{Si}_{3} \mathrm{~N}_{4}$ microresonators is $\mathcal{O}(1 \mathrm{GHz})$, while that in $\mathrm{MgF}_{2}$ resonators is $\mathcal{O}(1 \mathrm{MHz})$. Second, after each soliton switching the $\mathrm{MgF}_{2}$ resonator shows slower recoil of the $\mathcal{C}$-resonance than the $\mathrm{Si}_{3} \mathrm{~N}_{4}$ microresonator. This is attributed to the distinct thermal relaxation of the two platforms. The $\mathrm{MgF}_{2}$ resonator has a larger effective mode volume and physical size than the chip-scale $\mathrm{Si}_{3} \mathrm{~N}_{4}$ micro-ring resonators such that the thermal relaxation time is longer. In the evolution of the modulation response of the $\mathrm{MgF}_{2}$ resonator (Fig $3(\mathrm{f})$ ), the recoil of the $\mathcal{C}$-resonance leaves curved trajectory while it is very abrupt in the $\mathrm{Si}_{3} \mathrm{~N}_{4}$ microresonator (Fig. $3(\mathrm{~b}))$.

The non-destructive soliton probing scheme combined with the backward tuning allows an understanding of the soliton switching dynamics in microresonators. The modulation response clearly predicts the switching and therefore provides a convenient tool to control the soliton states and induce switching on demand. In experiments, one can perform deterministic switching by tuning the pump frequency, while monitoring the effective laser frequency detuning revealed by the VNA response.

Thermally enabled transitions of soliton states. We attribute the successive soliton switching in backward tuning to the thermal nonlinearity of optical microresonators. Due to material absorption, the intracavity energy of a soliton state thermally shifts the cavity resonance via thermal expansion and thermal change of the refractive index: $\widetilde{\omega}_{0}=\omega_{0}-\Delta_{\mathrm{T}}$, where $\Delta_{\mathrm{T}}$ is the thermally induced resonance shift which is approximately (neglecting cross term) proportional to the energy of intracavity field:

$$
\Delta_{\mathrm{T}}(N) \propto E_{\mathcal{C}}+N \cdot E_{\mathcal{S}}
$$

where $E_{\mathcal{C}}$ is the energy of the $\mathcal{C}$ component, $E_{\mathcal{S}}$ is the energy of one soliton and $N$ the number of solitons. Thus, the effective detuning can be expressed as $2 \pi \delta_{\text {eff }}=\omega_{0}-\Delta_{\mathrm{T}}-\omega_{\mathrm{p}}$. Physically, the soliton switching occurs when the laser backward tuning reduces the detuning to the bifurcation point of the system. This boundary value can be identified from the VNA trace and is represented by the position of the $\mathcal{S}$-resonance. After the switching, one soliton is extinct which decreases the energy in the cavity, and thereby reduces the thermal shift $\Delta_{\mathrm{T}}$. This spontaneously stabilizes the system in a new soliton state, by effectively increasing the effective detuning. The process is reflected in the evolution 
a

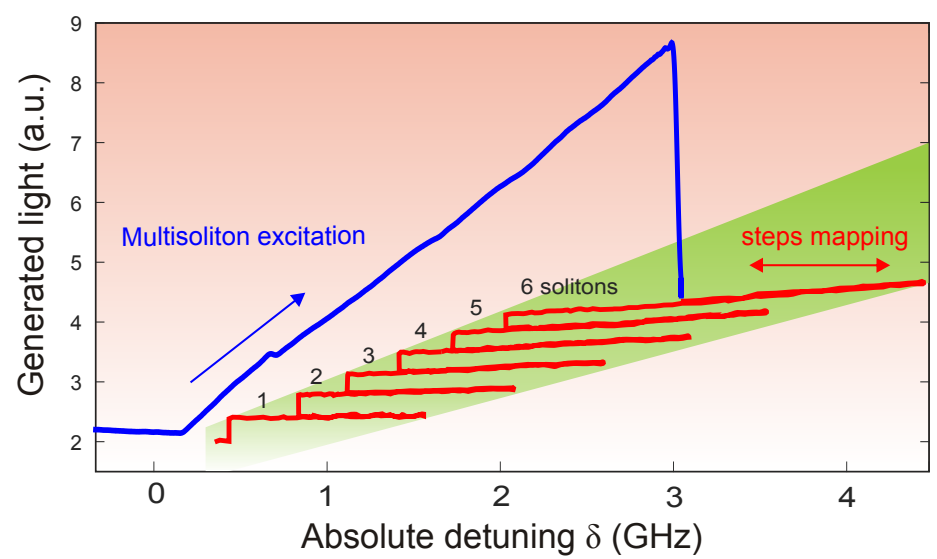

C

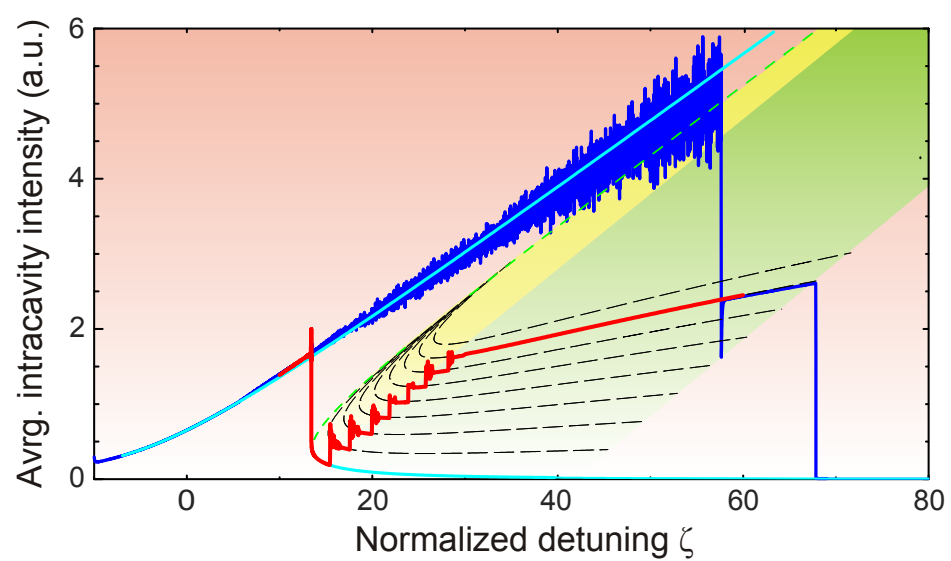

b

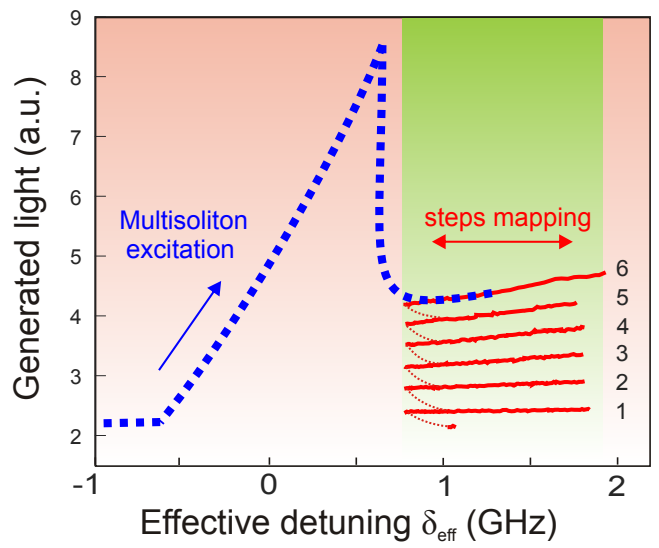

d

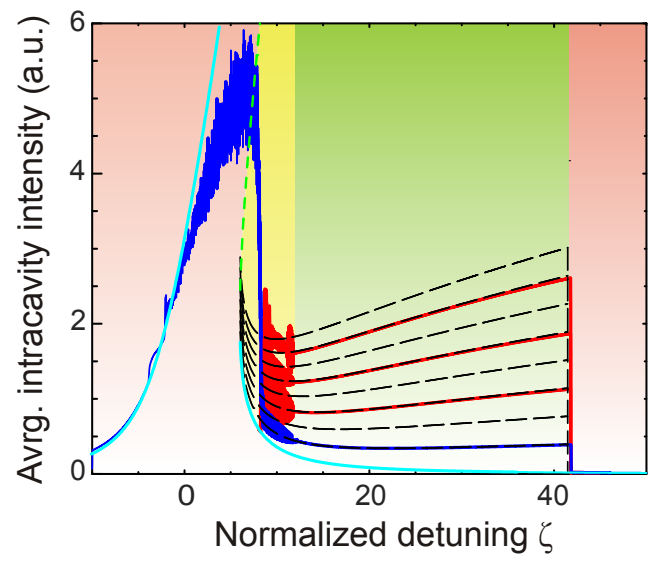

Figure 4. Experimental mapping of soliton existence range and numerical simulations. (a) Experimental measurements of the generated comb light with respect to the absolute detuning. The blue curve shows the trace in the forward tuning. The red curve indicates the entire soliton existence range. Zero absolute detuning corresponds to $\sim 1553.4 \mathrm{~nm}$. (b) Experimental trace from (a) plotted in terms of the effective detuning measured from the modulation response with the VNA. Hypothetical trace of forward tuning is shown in blue dashed line, because the effective detuning in this process can not be reliably measured with the VNA. (c, d) Numerical simulations and analytical solutions of the backward tuning in $\mathrm{Si}_{3} \mathrm{~N}_{4}$ with (c) and without (d) thermal effects. Normalized detuning used in the simulation: $\zeta=2\left(\omega_{0}-\omega_{\mathrm{p}}\right) / \kappa$, where $\omega_{0}$ is the resonance frequency, $\omega_{\mathrm{p}}$ is the pump frequency and $\kappa$ is the resonance linewidth. Blue lines indicate initial excitation of a multiple-soliton state in the forward tuning. Red lines indicate the backward tuning. Light blue lines indicate the stable branch of the nonlinear induced tilted resonance (in the CW mode). Dashed green lines indicate the unstable branch. The yellow area allows for the formation of breathing soliton states. The green area indicates the formation of stable solitons. Solitons cannot exist in red area. The dashed lines show analytical description of soliton steps, with analytical solution of soliton states in the system.

of the modulation response (see Fig $3(\mathrm{~b}))$ as a separation of $\mathcal{C}$ - and $\mathcal{S}$-resonance after the switching. It should be also noted that the recoiled $\mathcal{C}$-resonance frequency is similar after each switching event, because the resonator loses approximately similar amount of energy. Overall, the thermal nonlinearity lifts the degeneracy of soliton states with respect to the pump frequency.

Full mapping of the soliton induced multistability diagram in optical microresonator. The pump backward tuning enables deterministic and successive soliton switching, opening access to soliton states $N, N-1, \ldots, 1$. It is therefore possible to experimentally explore the soliton existence range in terms of the abso- lute and the effective detuning in each state, which to authors best knowledge has never been directly experimentally measured for cavity solitons of any kind. In terms of effective detuning, we express the soliton existence range as $\delta_{\mathrm{s}}<\delta_{\text {eff }}<\delta_{\max }$. The lower boundary $\delta_{\mathrm{s}}$ is identified in the backward soliton switching: it corresponds to the frequency where the $\mathcal{C}$-resonance and the fixed $\mathcal{S}$-resonance overlap. In the studied $\mathrm{Si}_{3} \mathrm{~N}_{4}$ microresonator under chosen pumping conditions this quantity is measured as $\delta_{\mathrm{s}} \sim 0.78 \mathrm{GHz}$. The upper detuning boundary $\delta_{\max }$ of the soliton existence range can be explored for each soliton state when the pump laser is tuned forward until the soliton comb disappears. Based on the theory 
and standard LLE simulations, this detuning is expected to be identical for all states corresponding to different number of solitons [10 (see Fig,4(d) and also SI), as the boundary of the energy balance of dissipative Kerr solitons. In experiments under the same pumping conditions such maximum effective detuning $\delta_{\max }$ is found for all soliton states as $\sim 2.0 \mathrm{GHz}$, yet no clear feature in the modulation response enables to predict this maximum boundary.

Figure 4(a) displays a one-trace mapping of six steps of soliton states in $\mathrm{Si}_{3} \mathrm{~N}_{4}$ microresonator as a function of the absolute pump frequency (wavelength) (i.e. the absolute detuning $\delta$ ). For each soliton step, we first tune the pump forward approaching the maximum detuning $\left(\delta \rightarrow \delta_{\max }\right)$, and then tune backward towards the soliton switching point $\left(\delta \rightarrow \delta_{\mathrm{s}}\right)$ where the soliton state is switched from $N$ to $N-1$. Since the thermally induced cavity resonance shift is included in the absolute frequency detuning, we observe that the soliton existence range in the absolute detuning is increasingly offset for a larger number of soliton. This creates the staircase pattern of the generated light and enables successive soliton switching. However, if the generated light trace is plotted with respect to the effective laser detuning $\left(\delta_{\text {eff }}\right)$ as done in Fig $4(\mathrm{~b})$, it appears that all the soliton steps are stacked vertically within the range $\delta_{\mathrm{s}}<\delta_{\text {eff }}<\delta_{\max }$, which corresponds to the expected theoretical diagram when the thermal effect is neglected [10].

We performed numerical simulations based on both LLE and coupled mode equations with the additional thermal relaxation equation included (cf. SI) which verify that the deterministic soliton switching is enabled by the thermal nonlinearity of the microresonator (Fig. 4 (c, d)). By including the thermal effects into numerical simulations, we are able to reproduce the staircase power trace, corresponding to the successive reduction of the soliton number in the backward pump tuning (cf. red curve in Fig 4(c)). Analytical power traces of soliton steps (black dashed lines) indicate soliton existence ranges for multiple-soliton states with different $N$. They reveal a displacement of the soliton existence range between different soliton states (qualitatively similar to the measured in Fig, $4(\mathrm{a})$ ) as a consequence of the thermal nonlinearity.

When the thermal effects in simulations are "switched off", soliton steps are well aligned and the soliton existence range is again degenerate with respect to the soliton number $(N)$, see Fig, 4(d). No soliton switching is therefore observed in the backward tuning. Numerical simulation also revealed the soliton breather states that is considered as a intermediate state between the chaotic MI operation regime and the stable soliton state. In the breather state, the soliton pulse peak power and the pulse duration, as well as the average intacavity energy, will experience periodical oscillations. This induces thermal perturbations to the cavity resonance and initiates the soliton switching.

\section{DISCUSSION}

We experimentally, numerically and analytically demonstrate the discovery that soliton states in a microresonator are not detuning degenerate, and can be individually addressed by laser detuning. We demonstrate that this effect is platform independent and can be used in a laser backward tuning process to achieve a successive reduction of the soliton number $(N \rightarrow N-1 \rightarrow \ldots \rightarrow 1)$. This deterministic switching is enabled by the thermal nonlinearity of the microresonator and provides a route to obtain a single soliton state from an arbitrary multiplesoliton state. We have shown non-destructive soliton probing technique, which enables to track the thermal impact of external perturbations of the system on its stability. The technique also allows to lock the soliton state against the impact of these perturbations and gives a clear insights of soliton dynamics inside the cavity. Combining this technique with the laser backward tuning allows for deterministic soliton switching and makes accessible any target multiple-soliton state in a predictable way. The results are in good agreement with analytical treatment of the soliton comb including thermal effects as well as numerical simulations, and can be applied to all Kerr nonlinear microresonators.

\section{METHODS}

$\mathrm{Si}_{3} \mathrm{~N}_{4}$ microresonators investigated in this work were fabricated using the Photonic Damascene process [38]. Microresonators have FSR of $100 \mathrm{GHz}$. A single mode "filtering" section was added to the micro-rings in order to suppress high-order modes [39]. The dispersion parameters of the microresonators are measured using the frequency comb assisted laser spectroscopy method 40]: $\frac{D_{2}}{2 \pi}=1-2 \mathrm{MHz}, \frac{D_{3}}{2 \pi}=\mathcal{O}(1 \mathrm{kHz})$ (where the resonance frequencies near $\omega_{0}$ are expressed in a series $\omega_{\mu}=\omega_{0}+\sum_{i \geq 1} D_{i} \mu^{i} / i$ !, where $i \in \mathbb{N}, \mu \in \mathbb{Z}$ is the mode number). Pumped resonance is at $1553.4 \mathrm{~nm}$. Tuning speed for soft excitation is $\sim 1 \mathrm{~nm} / \mathrm{s}$. Pump power is $\sim$ $2-3 \mathrm{~W}$ on a chip.

The detailed scheme of the experimental setup is presented in the Figure 2(a). The $\mathrm{Si}_{3} \mathrm{~N}_{4}$ resonator is pumped with a CW laser light from an external-cavity diode laser amplified by an erbium-doped fiber amplifier (EDFA) to $3-5 \mathrm{~W}$. The $\mathrm{CW}$ pump is coupled to the onchip resonator using lensed fibers with coupling losses of $2.5-3 \mathrm{~dB}$ per facet. For the soliton probing measurements $10 \mathrm{GHz}$ electro-optical phase modulator (EOM) is placed before EDFA with additional polarization controller for adjusting input polarization. The pump frequency wavelength in the pump backward tuning is mea- 
sured by a wave-meter with resolution of $\sim 50 \mathrm{MHz}$. For the long sweeps an arbitrary function generator is used. The output signal from the chip is split in several paths among OSA (for the measurements of combs spectra), oscilloscope (for the measurements of generated light by filtering out the pump with FBG) and a VNA receiver (for the measurements of and modulation response).

The $\mathrm{MgF}_{2}$ crystalline resonator was fabricated by diamond turning of a cylinder blank and subsequent hand polishing to achieve high $Q$ (linewidth $\frac{\kappa}{2 \pi}=100 \mathrm{kHz}$ ). The diameter of $5 \mathrm{~mm}$ yields a FSR $\frac{D_{1}}{2 \pi}=14 \mathrm{GHz}$. The dispersion parameters at the pump wavelength of 1553 $\mathrm{nm}$ are: $\frac{D_{2}}{2 \pi}=1.9 \mathrm{kHz}, \frac{D_{3}}{2 \pi}=\mathcal{O}(1 \mathrm{~Hz})$. The pump laser (fiber laser, wavelength $1553 \mathrm{~nm}$; short-term linewidth $10 \mathrm{kHz}$ ) is amplified to $\sim 250 \mathrm{~mW}$. The relative laser frequency is monitored by counting the heterodyne beat between the pump laser and a reference laser stabilized to an ultra-stable cavity. The light is evanescently coupled to a WGM with a tapered optical fiber.

* M.K., H.G. and E.L. contributed equally to this work

† tobias.kippenberg@epfl.ch

[1] P. Del'Haye, A. Schliesser, O. Arcizet, T. Wilken, R. Holzwarth, and T. J. Kippenberg, Nature 450, 1214 (2007)

[2] T. J. Kippenberg, R. Holzwarth, and S. Diddams, Science 332, 555 (2011).

[3] T. J. Kippenberg, S. M. Spillane, and K. J. Vahala, Phys. Rev. Lett. 93, 083904 (2004)

[4] A. A. Savchenkov, A. B. Matsko, D. Strekalov, M. Mohageg, V. S. Ilchenko, and L. Maleki, Phys. Rev. Lett. 93, 243905 (2004).

[5] P. Del'Haye, T. Herr, E. Gavartin, M. L. Gorodetsky, R. Holzwarth, and T. J. Kippenberg, Phys. Rev. Lett. 107, 063901 (2011)

[6] Y. Okawachi, K. Saha, J. Levy, H. Wen, M. Lipson, and A. Gaeta, Opt. Lett. 36, 3398 (2011).

[7] T. Herr, K. Hartinger, J. Riemensberger, C. Wang, E. Gavartin, R. Holzwarth, M. L. Gorodetsky, and T. J. Kippenberg, Nat. Photon. 6, 480 (2012).

[8] F. Ferdous, H. Miao, D. E. Leaird, K. Srinivasan, J. Wang, L. Chen, L. T. Varghese, and A. M. Weiner, Nature Photon. 5, 770 (2011).

[9] S. Papp, P. Del'Haye, and S. Diddams, Opt. Express 21, $17615(2013)$

[10] T. Herr, V. Brasch, J. D. Jost, C. Wang, M. Kondratiev, M. L. Gorodetsky, and T. J. Kippenberg, Nat. Photon. 8, 145 (2014).

[11] V. Brasch, M. Geiselmann, T. Herr, G. Lihachev, M. H. P. Pfeiffer, M. L. Gorodetsky, and T. J. Kippenberg, Science (2015), 10.1126/science.aad4811.

[12] M. Karpov, H. Guo, A. Kordts, V. Brasch, M. H. P. Pfeiffer, M. Zervas, M. Geiselmann, and T. J. Kippenberg, arXiv:1506.08767 (2015).

[13] X. Yi, Q.-F. Yang, K. Y. Yang, M.-G. Suh, and K. Vahala, Optica 2, 1078 (2015).

[14] T. Ideguchi, S. Holzner, B. Bernhardt, G. Guelachvili, N. Picqué, and T. W. Hänsch, Nature 502, 355 (2013).
[15] J. Pfeifle, V. Brasch, M. Lauermann, Y. Yu, D. Wegner, T. Herr, K. Hartinger, P. Schindler, J. Li, D. Hillerkuss, R. Schmogrow, C. Weimann, R. Holzwarth, W. Freude, J. Leuthold, T. J. Kippenberg, and C. Koos, Nat. Photon. 8, 375 (2014)

[16] J. Pfeifle, A. Kordts, P. Marin, M. Karpov, M. H. P. Pfeiffer, V. Brasch, R. Rosenberger, J. Kemal, S. Wolf, W. Freude, T. J. Kippenberg, and C. Koos, in CLEO: 2015 Postdeadline Paper Digest (Optical Society of America, 2015) p. JTh5C.8.

[17] S. Papp, K. Beha, P. Del'Haye, F. Quinlan, H. Lee, K. Vahala, and S. Diddams, Optica 1, 10 (2014).

[18] F. Leo, S. Coen, P. Kockaert, S.-P. Gorza, P. Emplit, and M. Haelterman, Nat. Photon. 4, 471 (2010).

[19] W. Liang, D. Eliyahu, V. Ilchenko, A. Savchenkov, A. Matsko, D. Seidel, and L. Maleki, Nat. Commun. 6 (2015).

[20] I. S. Grudinin and N. Yu, in Laser Resonators, Microresonators, and Beam Control XVII, Proceedings of SPIE, Vol. 9343 (2015) pp. 93430F-93430F-9, $10.1117 / 12.2085420$.

[21] J. K. Jang, M. Erkintalo, S. Coen, and S. G. Murdoch, Nature communications 6 (2015).

[22] Y. K. Chembo and N. Yu, Phys. Rev. A 82, 033801 (2010).

[23] Y. Chembo and C. Menyuk, Phys. Rev. A 87, 053852 (2013).

[24] S. Coen, H. Randle, T. Sylvestre, and M. Erkintalo, Opt. Lett. 38, 37 (2013)

[25] C. Godey, I. Balakireva, A. Coillet, and Y. Chembo, Phys. Rev. A 89, 063814 (2014).

[26] M. Lamont, Y. Okawachi, and A. Gaeta, Opt. Lett. 38, 3478 (2013).

[27] J. Levy, A. Gondarenko, M. Foster, A. Turner-Foster, A. Gaeta, and M. Lipson, Nat. Photon. 4, 37 (2010)

[28] D. Moss, R. Morandotti, L. Gaeta, and M. Lipson, Nat. Photon. 7, 597 (2013)

[29] M. A. Foster, J. S. Levy, O. Kuzucu, K. Saha, M. Lipson, and A. L. Gaeta, Opt. Express 19, 14233 (2011)

[30] V. S. Ilchenko, A. A. Savchenkov, A. B. Matsko, and L. Maleki, Phys. Rev. Lett. 92, 043903 (2004).

[31] I. S. Grudinin, A. B. Matsko, A. A. Savchenkov, D. Strekalov, V. S. Ilchenko, and L. Maleki, Opt. Commun. 265, 33 (2006).

[32] W. Liang, A. A. Savchenkov, A. B. Matsko, V. S. Ilchenko, D. Seidel, and L. Maleki, Opt. Lett. 36, 2290 (2011)

33] V. B. Braginsky, M. L. Gorodetsky, and V. S. Ilchenko, Physics Letters A 137, 393 (1989).

[34] T. Carmon, L. Yang, and K. Vahala, Opt. Express 12, $4742(2004)$

[35] X. Yi, Q.-F. Yang, K. Y. Yang, M.-G. Suh, and K. Vahala, Optica 2, 1078 (2015)

[36] A. E. Fomin, M. L. Gorodetsky, I. S. Grudinin, and V. S. Ilchenko, J. Opt. Soc. Am. B 22, 459 (2005).

[37] A. B. Matsko and L. Maleki, Phys. Rev. A 91, 013831 (2015)

[38] M. H. P. Pfeiffer, A. Kordts, V. Brasch, M. Zervas, M. Geiselmann, J. D. Jost, and T. J. Kippenberg, Optica 3, 20 (2016)

[39] A. Kordts, M. Pfeiffer, H. Guo, V. Brasch, and T. J. Kippenberg, arXiv:1511.05381 (2015).

[40] P. Del'Haye, O. Arcizet, M. L. Gorodetsky, R. Holzwarth, and T. J. Kippenberg, Nat. Photon. 3, 529 (2009) 


\section{ACKNOWLEDGEMENTS}

Acknowledgements. This publication was supported by Swiss National Science Foundation (SNF) as well as Contract W31P4Q-14-C-0050 from the Defense Advanced Research Projects Agency (DARPA), Defense Sciences Office (DSO). This material is based upon work supported by the Air Force Office of Scientific Research, Air Force Material Command, USAF under Award No. FA9550-15-1-0099. This publication was supported by funding from the European Space Agency (ESA), European Space Research and Technology Centre (ESTEC). G.L., V.E.L. and M.L.G. were supported by the Ministry of Education and Science of the Russian Federation project \#4.585.21.0005. The authors gratefully acknowledge valuable discussions with Michael Geiselmann, Martin H.P. Pfeiffer, John D. Jost and Victor Brasch. All samples were fabricated and grown in the Center of MicroNanoTechnology (CMi) at EPFL.

\section{AUTHORS CONTRIBUTIONS}

M.K. designed and performed the experiments and analyzed the data. H.G. conceived and initiated the numerical simulations. E.L. performed experiments in $\mathrm{MgF}_{2}$ microresonators and analyzed the data. A.K. fabricated the $\mathrm{Si}_{3} \mathrm{~N}_{4}$ samples and M.P. developed the fabrication method. G.L. and V.E.L. assisted in simulations. M.L.G. developed the theory and performed the simulations. M.K., H.G., E.L., M.L.G., T.J.K. discussed all data in the manuscript. M.K. and H.G. wrote the manuscript, with input of E.L., M.L.G., T.J.K. T.J.K. supervised the project. 